\title{
Endodontic emergencies at the Yalgado Ouedraogo University Hospital Center: Prevalence, epidemiological, and therapeutic aspects
}

\author{
(1) Wendpoulomdé Aimé Désiré Kaboré, ${ }^{1}$ (1) Diouma Ndiaye, ${ }^{2}$ (1) Seydina Ousmane Niang, ${ }^{2}$ \\ Anta Seck, ${ }^{2}$ (1) Marie Chantal Avoaka-Boni, ${ }^{3}$ (ㄷ) Khaly Bane ${ }^{2}$ \\ 'Department of Conservative Dentistry and Endodontics/Training and Research Unit in Health Sciences, \\ Joseph KI-ZERBO University, Ouagadougou, Burkina Faso \\ ${ }^{2}$ Conservative Dentistry and Endodontics Service/Cheikh Anta Diop Dakar University, Dakar-fann, Senegal \\ ${ }^{3}$ Department of Conservative Dentistry and Endodontics, Felix Houphouët Boigny University, Abidjan, Ivory Coast
}

\begin{abstract}
Purpose: Endodontic emergencies constitute a substantial portion of dental emergencies. This study was carried out at the Yalgado Ouédraogo University Hospital Center aimed to determine the different types of endodontic emergencies and their epidemiological and therapeutic aspects to better understand these events.
\end{abstract}

Methods: This was a descriptive, retrospective, cross-sectional study conducted from January 1, 2017, to December 31, 2017, involving patients who sought an emergency consultation. The collected variables were age, gender, the reason for consultation, diagnosis, and treatment.

Results: The number of included patients was 227, of whom 126 (56.8\%) were female. Endodontic emergency consultations accounted for $51.54 \%$ of the dental emergencies. Pain was the main reason for the emergency consultation in $60 \%$ of cases. Irreversible pulpitis was the most common type of endodontic emergency, with a frequency of $44.8 \%$. The most common (55.5\%) emergency treatment was based on medication followed by a surgical treatment (22.47\%) and medical-surgical treatment (22.03\%).

Conclusion: This study showed that endodontic emergencies are the most common type of dental emergency. The lack of equipment and medical consumables limits the quality of care for such cases.

Keywords: Endodontic emergency, epidemiology, prevalence, treatment.

\section{Introduction}

An emergency situation is a sudden or unexpected threat to one's well-being or one's physical integrity (1). An emergency in dentistry most often involves acute and uncontrolled pain for the patient that requires immediate intervention by a dental surgeon (2). These emergencies can be due to infectious and/or inflammatory pathologies and trauma involving the oral-dental structures, and rarely life threatening $(3,4)$. Minimal access to dental health services or negligence of the dental cause by the patient can be the underlying causes (4). In a study of oral-dental health in the globally, Marcenes et al. (2013) highlighted the need

Cite this article as: Kaboré WAD, Ndiaye D, Niang SO, Seck A, Avoaka-Boni MC, Bane K. Endodontic emergencies at the Yalgado Ouedraogo University Hospital Center: Prevalence, epidemiological, and therapeutic aspects. Turk Endod J 2021;6:19-23.

Correspondence: Wendpoulomdé Aimé Désiré Kaboré. Unité De Formation Et De Recherches En Sciences De La Santé (ufr/ sds)/Université Joseph KI-ZERBO, 03 Bp 7021 Ouagadougou 03, Burkina Faso

Tel: +22625333434 e-mail:dr_kabore@yahoo.fr

Submitted: February 19, 2021 Accepted: April 19, 2021

(C)2021 Turkish Endodontic Society 
for an urgent response due to the increase in dental pathologies in most African countries (5). Indeed, the availability and accessibility of dental services are extremely limited in many African countries, delaying the consultation and increasing the number of emergency situations (6). In Burkina Faso, oral-dental afflictions are very common in the general population. A study in 2015 in Ouagadougou showed that there was a high need for oral health care although it was not sufficiently available (7). Endodontic emergencies constitute a substantial part of odontological emergencies. They account for approximately $90 \%$ of all dental consultations (8). Kaboré et al. (2016) reported in a study in Ouagadougou that the requests for consultation specifically involved conservative endodontics in $54.5 \%$ of the cases (9). The Yalgado Ouédraogo University Hospital Center (YO-UHC) is the main national hospital center of Burkina Faso. It comprises a stomatology, maxillofacial surgery, and odontology emergency unit (UUOSCMF). Therefore, it seemed relevant to carry out a study to determine the various types of endodontic emergencies, and their epidemiological and therapeutic aspects, to have a better understanding of these events.

\section{Materials and Methods}

\section{Setting, Type, and Timing of the Study}

This study was carried out at the Yalgado Ouédraogo University Hospital Center in the Department of Odontology, Stomatology, and Maxillofacial Surgery. It was a descriptive, retrospective, cross-sectional study, from January 1 , 2017, to December 31, 2017, regarding all of the files of patients seen for an emergency consultation, at the UUOSCMF of the YO-UHC.

\section{Criteria for Inclusion and Exclusion}

The study population comprised all of the patients of any age who consulted for an emergency at the UUOSCMF during the period of the study. This study included all of the patients who consulted for an odontological emergency and who received emergency treatment. The variables collected were age, gender, the reason for the consultation, the diagnosis, and the therapeutic procedure carried out. All of the patients who had a complete or useable clinical file were included in the study. Patients whose files did not contain the parameters of our study were not included in the study.

\section{Collection of the Data}

The technique for collecting the data consisted of analysis of the content of the registers and the consultation forms during the period of the study. An individual survey form was used to compile the data. This retrospective analysis of the registers and the consultation forms was carried out by the same operator so as to minimize the risks of error. The following variables were collected:

- The sociodemographic variables (age, gender, level of education, place of residence, and profession)

- The type of emergency: Endodontic (reversible and irreversible pulpitis, apical periodontitis, acute alveolar abscesses, and endodontic flare-ups), locoregional infections (cellulitis and alveolitis), periodontal (septum syndrome, pericoronitis, gum abscesses, periodontal abscesses, and necrotizing periodontal diseases), traumatic (involving the dental tissues, the support tissues, as well as the soft tissues), hemorrhagic emergencies (post-operative or spontaneous oral hemorrhages), prosthetic emergencies (loosening of temporary or permanent crowns, fractures of adjoining prostheses, and prosthetic lesions), orthodontic emergencies (injury due to an orthodontic device, loosening of a casing), and the other types of emergencies (emergencies linked to the manducatory tract, restorative odontology, and facial pains)

- The therapeutic variables: The medical and/or the surgical treatment.

\section{Analysis of the Data}

The collected data were entered into a microcomputer using Microsoft Office Word 2013 software. The treatment and the analysis of the collected data were performed with the English version of EPI-info 7.1.3.3 statistics software. The graphs were created with Excel 2013 software.

\section{Ethics Considerations}

The study protocol has been approved by the hospital direction (Authorization $\mathrm{N}^{\circ} 0008 / \mathrm{MS} / \mathrm{SG} / \mathrm{CHU}-\mathrm{YO} /$ DG issued on January 9,2018 ). The anonymity of the collected files as well as the confidentiality of the content was respected.

\section{Results}

\section{Characteristics of the Patients}

From January 1, 2017 to December 31, 2017, that is, a period of 12 months, 760 patients consulted for an odontological emergency. There were 227 complete and retained files, or a monthly incidence of 18.9 cases (Figure 1). Men represented 98 cases (43.2\%) and women 129 cases (56.8\%), or a sex ratio of 0.78 . The age of the patients varied from 2 years to 73 years, with an average of 29 years \pm 31.67 years. 
The 19-40 years of age bracket were represented the most, with 127 patients, or $56 \%$. Most of the sample (71.4\%) was represented by patients with low incomes (casual workers, pupils, students, employed, and homemakers). The patients lived in urban areas in $90.8 \%$ of cases, a semi-urban area in $5.3 \%$ of cases, and a rural area in $3.9 \%$ of cases.

\section{Repartition of the Types of Emergencies Encountered}

Endodontic emergency consultations comprised 116, or $51.54 \%$, of the cases of odontological emergencies (Figure 2).

\section{The Types of Endodontic Emergencies Encountered}

Pain was the main reason for the emergency consultation in $60 \%$ of cases. Irreversible pulpitis was by far the most often encountered type of emergency, with an incidence of $44.8 \%$ (Table 1 ).

\section{Therapeutic Aspects}

The prescription of medications was the most common emergency therapeutic $(55.5 \%)$ followed by surgical treat-

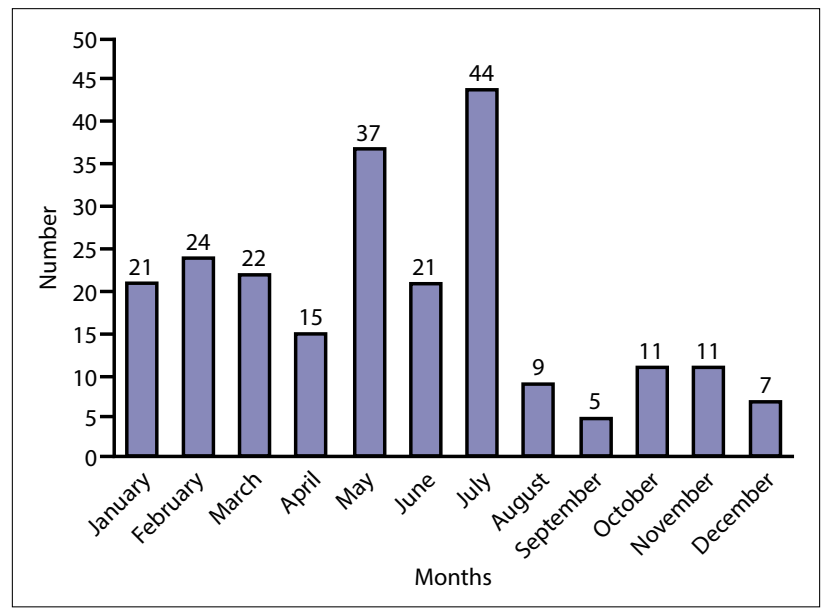

Fig. 1. Monthly repartition of the emergency consultations.

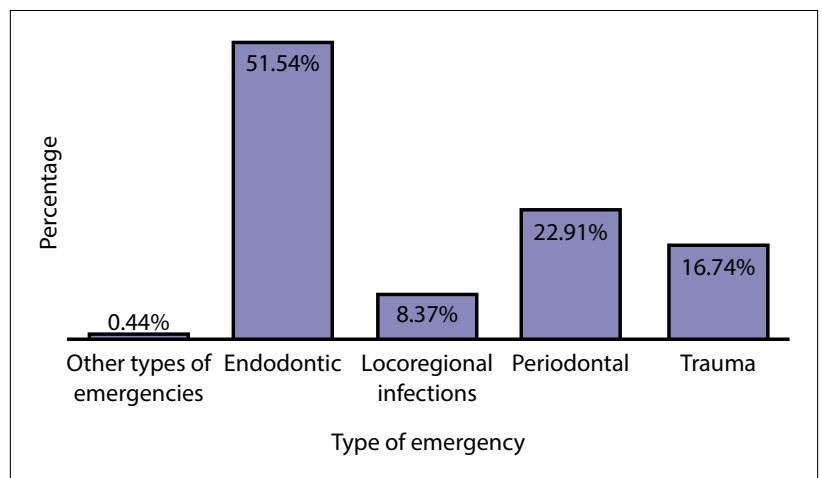

Fig. 2. Repartition of the types of emergencies identified. ment $(22.5 \%)$ and medical-surgical treatment $(22 \%)$. There were 165 prescriptions for analgesia and 126 for antibiotics. Paracetamol was the analgesic used the most (67.9\%) (Table 2). Amoxicillin was prescribed in $39.7 \%$ of the cases of antibiotic prescription (Table 3 ). There were 31 patients, or $13.7 \%$, who received an adjuvant treatment (mouthwash). One hundred and one therapeutic procedures were undertaken. Tooth extraction (36.6\%) was the most frequently performed surgical treatment performed followed by indirect pulp capping (28.7\%) (Table 4).

Table 1. Distribution of the endodontic emergencies $(n=116)$

\begin{tabular}{lcc} 
Endodontic emergencies & Number (n) & Percentage \\
\hline Irreversible pulpitis & 52 & 44.8 \\
Reversible pulpitis & 31 & 26.7 \\
Acute alveolar abscess & 24 & 20.7 \\
Acute apical periodontitis & 9 & 7.8 \\
Total $n$ (\%) & 116 & 100 \\
\hline
\end{tabular}

Table 2. Repartition of the patients according to the analgesic used $(n=165)$

\begin{tabular}{lcc} 
Analgesic & Frequency (n) & Percentage \\
\hline Paracetamol & 112 & 67.9 \\
Paracetamol + Codeine & 26 & 15.7 \\
Nonsteroidal anti-inflammatory drugs & 14 & 8.5 \\
Steroidal anti-inflammatories & 13 & 7.9 \\
Total n (\%) & 165 & 100 \\
\hline
\end{tabular}

Table 3. Repartition of the patients according to the antibiotic used

\begin{tabular}{lcc} 
Antibiotic & Frequency (n) & Percentage \\
\hline Amoxicillin & 50 & 39.7 \\
Amoxicillin + Metronidazole & 37 & 29.4 \\
Amoxicillin + Clavulanic acid & 30 & 23.8 \\
Lincomycin & 9 & 7.1 \\
Total $\mathrm{n}(\%)$ & 126 & 100 \\
\hline
\end{tabular}

Table 4. Repartition of the surgical treatments $(n=101)$

\begin{tabular}{lcc} 
Surgical treatment & Number $(\mathbf{n})$ & Percentage \\
\hline Dental avulsion & 37 & 36.6 \\
Indirect pulp capping & 29 & 28.7 \\
Pulpectomy & 9 & 8.9 \\
Extraction of a loose tooth fragment & 7 & 6.9 \\
Canal medication & 6 & 6 \\
Mucous cap removal & 5 & 4.9 \\
Transcanal drainage & 4 & 4 \\
Disocclusion & 2 & 2 \\
Drainage by incision & 1 & 1 \\
Retainer & 1 & 1 \\
Total $n$ (\%) & 101 & 100 \\
\hline
\end{tabular}




\section{Discussion}

During the 12 -month period of the study, there were 227 cases of dental emergencies or a monthly incidence of 18.9 cases per month. Over a period of 1 month in 2016, Kaboré et al. in Burkina Faso reported 200 cases of emergency consultations at the Municipal Health Center (9). Popescu et al. (2015) in France reported a monthly incidence of $78.5 \%$ cases in a study of dental emergencies from January to December 2013 (10). The results of our study could be explained by the limited availability of consumables and technical failures (malfunction of the dental chair or rotary instruments) frequently observed at the UUOSCMF. Similarly, the strong influence of traditional medicine and recourse to self-medication significantly contributes to the limitation of hospital visits $(8,11)$. Female patients represented $56.8 \%$ of cases, with a sex ratio of 0.78. Studies in Burkina have made the same findings $(12,13)$. However, in 2015, Popescu et al. reported that the majority of patients in France were male (10). The average age was 29 years, with extremes of 2 years and 73 years. The most represented age bracket was from 19 to 40 years $(55.95 \%)$. Other studies have found a high prevalence of the same $20-40$ years age bracket, at $52.5 \%$ and $55 \%(9,14)$. Most of the sample $(71.4 \%)$ was represented by patients with low incomes. The same finding was noted in the series by Kaboré et al. (2016) in Burkina Faso (9). Moreover, Jaiswal et al. (2015) in India reported that disadvantaged populations were more affected by dental pathologies (15). Endodontic emergencies were by far the most encountered type of emergency, with a frequency of $51.54 \%$. Our results are in keeping with other African and international studies $(14,16)$. In the present study, the most represented endodontic emergency was irreversible pulpitis $(44.8 \%)$. This was followed by reversible pulpitis $(26.7 \%)$, acute alveolar abscess $(20.7 \%)$, and acute apical periodontitis (7.8\%). Tamietti et al. (2012) obtained similar results in a study carried out in the odontological emergency services in Brazil (17). They found that pulpitis followed by periapical abscesses were the causes for consultation. However, for Touré et al. (2011), acute apical periodontitis was the main pathology in endodontic emergencies (16). In 2011, Varenne et al. in Ouagadougou reported that the most common reasons for a dental consultation were cavities with pulp involvement (52.4\%) (18). The most frequently performed emergency treatment in UUOSCMF was prescription of medications only (55.5\%). A therapeutic procedure was undertaken in $22.5 \%$ of cases and a medical-surgical treatment in $22 \%$ of cases. These results are similar to those of a recent study in Burkina Faso that found a predominance of medication prescription (8). In a 2014 study of the treatment of toothaches in preschool-age children in Brazil, Machado et al. made the same finding, with $87 \%$ of the cases treated by prescription of medication (19). Analgesic treatment, paracetamol in this case, was the most often prescribed medical treatment $(67.9 \%)$ followed by antibiotic therapy based on amoxicillin (39.7\%). In 2016, Kaboré et al. in Burkina Faso reported that $45.5 \%$ of the medication prescriptions were based on an association of amoxicillinmetronidazole-paracetamol (7). Tooth extraction (36.6\%) was the most often performed surgical procedure followed by indirect capping (28.7\%). These results are comparable to those of Ouédraogo et al. (2011) in Burkina Faso, who also found a predominance of dental avulsions (47.81\%) and of conservative care (38.69\%) (4). This high frequency of dental avulsions may be explained by the inadequate technical platform and the lack of resources at the UUOSCMF to perform endodontic treatment. Due to this general trend, developing countries have not benefited significantly from the tremendous progress in dental care that allows a tooth that is infected or even decayed to be treated and fully restored instead of having to resort to extraction. The high prevalence of tooth cavities in African countries, particularly in urban areas, can be associated with changes in dietary habits, or to negligence and/or unawareness of the risk factors for oral-dental diseases and of their consequences. A study carried out in Burkina Faso reported that $75.8 \%$ of Burkinabe dental surgeons wrote a prescription after indirect capping (20). The prescription by the dental surgeon is most often issued in the context of pain or an obvious or predictable infection following an intervention by them (in prophylaxis). However, most endodontic emergencies require a therapeutic procedure. Medication prescription alone is not relevant.

\section{Conclusion}

In Burkina Faso, endodontic emergencies are a real challenge that needs to be recognized. Indeed, for odontologists, these emergencies are not the exception but a common occurrence in daily practice. It is, therefore, paramount that a serious effort is made to assess how the numerous obstacles can be resolved to be able to provide the best possible appropriate therapeutic. This study has shown that endodontic emergencies are the most often encountered type of odontological emergency, but that the lack of suitable equipment and the restricted availability of consumables contribute to limiting the quality of their treatment.

Authorship Contributions: Concept: W.A.D.K., K.B.; Design: W.A.D.K., S.O.N.; Supervision: K.B.; Materials: W.A.D.K.; Data: W.A.D.K.; Analysis: W.A.D.K., S.O.N.; Literature search: W.A.D.K., D.N.; Writing: W.A.D.K., D.N.; Critical revision: K.B., M.C.A.B., A.S. 
Acknowledgements: The author would like to thank the head of the stomatology, maxillofacial surgery and odontology emergency unit.

Source of Funding None declared.

Conflict of Interest $\$$ None declared.

Ethical Approval: The study protocol has been approved by the Ministeré De La Santé Sécretariat Général Centre Hospitalier Universitaire Yalgado Ouedraogo Direction Générale (date: January 9, 2018, protocol no: 0008/MS/SG/CHU$\mathrm{YO} / \mathrm{DG})$.

\section{References}

1. Morgans A, Burgess SJ. What is a health emergency? The difference in definition and understanding between patients and health professionals. Aust Health Rev 2011; 35 : 284-9. [CrossRef]

2. Guivarc'h M, Maille G, Bukiet F, Le Coz P. Emergency and after-hours care in dentistry: a need for an ethical consideration. Ethics, Medicine and Public Health 2017; 3: 460-4.

3. Arbab-Chirani R, Chevalier V, Roux M, Boisramé-Gastrin S, LAzou D, Colemard F. On call emergency dental hospital service: creation and preliminary results. [Article in French]. Rev Epidemiol Sante Publique 2010; 58: 217-24.

4. Lafon A, Larras P, Ahossi V. Odontological emergencies. EMC - Emergency Medicine 2017: 1-22.

5. Marcenes W, Kassebaum NJ, Bernabé E, et al. Global burden of oral conditions in 1990-2010: a systematic analysis. J Dent Res 2013; 92: 592-7. [CrossRef]

6. Hescot P, China E, Bourgeois D, et al. The FDI African strategy for oral health: addressing the specific needs of the continent. Int Dent J 2013; 63: 113-20. [CrossRef]

7. Kaboré WAD, Garé JV, Niang SO, Guiguimdé PWL, Ouédraogo Y, Rodriguez I. The oral-health care offer in urban areas in Burkina Faso: example from the city of Ouagadougou. Rev Iv Odonto-Stomatol 2015; 17: 42-9.

8. Kaboré WAD, Ouédraogo CDW, Konaté A, et al. Selfmedication in oral diseases in Ouagadougou, Burkina Faso. Med Buccale Chir Buccale 2016; 22: 277-84. [CrossRef]

9. Kaboré WAD, Ouédraogo CDW, Millogo M, Traoré AS, Barro N, Sangaré L. Reasons for dental consultation in Ouagadougou. Rev Iv Odonto-Stomatol 2016; 18: 21-7.

10. Popescu LD, Aga I, Popescu MA. Management of dental emergencies in the Centre Hospitalier MétropoleSavoie after one year of running an on-call duty line. J Oral Med Oral Surg 2015; 21: 211-8.

11. Guiguimdé WPL, Millogo M, Konsem T, Ouédraogo D. Accidents due to the development of the bottom wisdom tooth in the odonto-stomatology andmaxillofacial surgery department at yalgado ouedraogo teaching hospital. Med Buccale Chir Buccale 2015; 21:5-9. [CrossRef]

12. Ouedraogo Y, Kabore WA, Konsem T, et al. Tooth decay: epidemiological and therapeutic aspects in dental service of University Teaching Hospital Yalgado Ouedraogo and Municipal Centre of Oral Health. Odontostomatol Trop 2015; 38: 49-55.

13. Ouédraogo D, Millogo M, Konsem T, Assouan C, Tioye YL, Ouoba K. Oral health care offer at the Yalgado Ouedraogo University Hospital Center (YOUHC) in Ouagadougou. Rev Col Odonto Stomatol Afr Chir Maxillo-Fac 2011; 18: 10-3.

14. Demenech LS, da Silva BM, Vianna de Oliveira K, et al. Epidemiological data and relevance of care provided in dental emergency service of a private hospital. RSBO 2015; $12: 339-45$.

15. Jaiswal AK, Pachava S, Sanikommu S, Rawlani SS, Pydi S, Ghanta B. Dental pain and self-care: a cross-sectional study of people with low socio-economic status residing in rural India. Int Dent J 2015; 65: 256-60. [CrossRef]

16. Touré B, Azogui-Lévy S, Dzara I, Azerad I, Boucher Y. Qualities of endodontic treatments in a sample of patients consulting urgently in the dentistry department at GHPS. Rev Odont Stomat 2011; 40: 280-92.

17. Tamietti MB, Martins MAP, Abreu MHNG, De Castilho LS. Factors associated with self-medication in a Brazilian emergency dental service. Pesq Bras Odontoped Clin Integr João Pessoa 2012; 2: 65-9. [CrossRef]

18. Varenne B, Fournet F, Cadot E, et al. Family environment and dental health disparities among urban children in Burkina Faso. [Article in French]. Rev Epidemiol Sante Publique 2011; 59: 385-92. [CrossRef]

19. Machado GC, Daher A, Costa LR. Factors associated with no dental treatment in preschoolers with toothache: a cross-sectional study in outpatient public emergency services. Int J Environ Res Public Health 2014; 11: 8058-68.

20. Kaboré WAD, Faye B, Ouédraogo Y, Konsem T, Bane K, Sarr M. Level of knowledge and dispositions of dental surgeons in Burkina Faso regarding endodontic emergencies. Int J Dent Oral Health 2015; 2: 1-4. [CrossRef] 